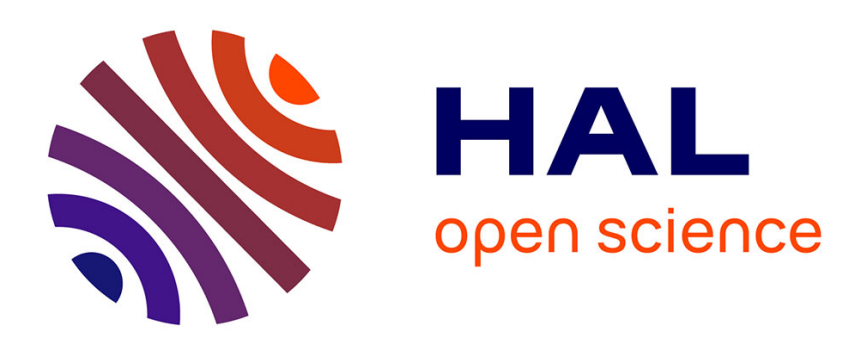

\title{
Analyse chimique d'un superalliage base nickel par microscopie électronique à haute résolution
}

\author{
J. Pénisson
}

\section{To cite this version:}

J. Pénisson. Analyse chimique d'un superalliage base nickel par microscopie électronique à haute résolution. Journal de Physique IV Proceedings, 1996, 06 (C2), pp.C2-117-C2-123. 10.1051/jp4:1996216 . jpa-00254194

\section{HAL Id: jpa-00254194 https://hal.science/jpa-00254194}

Submitted on 1 Jan 1996

HAL is a multi-disciplinary open access archive for the deposit and dissemination of scientific research documents, whether they are published or not. The documents may come from teaching and research institutions in France or abroad, or from public or private research centers.
L'archive ouverte pluridisciplinaire HAL, est destinée au dépôt et à la diffusion de documents scientifiques de niveau recherche, publiés ou non, émanant des établissements d'enseignement et de recherche français ou étrangers, des laboratoires publics ou privés. 


\title{
Analyse chimique d'un superalliage base nickel par microscopie électronique à haute résolution
}

\author{
J.M. Pénisson \\ CEA/Département de Recherche Fondamentale sur la Matière Condensée/SP2M/S, 17 rue des Martyrs, \\ 38054 Grenoble cedex 9, France
}

\begin{abstract}
The knowledge of the chemical composition at the atomic level of alloys is essential to understand their physical properties. A detailed analysis of the contrast of high resolution images can lead to a semi quantitative evaluation of local changes in the composition. The method will be applied to the study of the $\gamma / \gamma^{\prime}$ interface in a nickel based superalloy and to the determination of the distribution of heavy atoms in the $\gamma^{\prime}$ phase.
\end{abstract}

\section{INTRODUCTION}

Les alliages métalliques modernes ont la plupart du temps une composition très complexe et contiennent de nombreux constituants différents. Il est alors très important de connaitre avec un maximun de résolution la localisation de différents éléments au sein de l'alliage. Plusieurs techniques expérimentales telles que la sonde atomique [1], l'ALCHEMI [2] ou la cartographie de pertes d'énergie [3] peuvent être employées. Dans cet article, une méthode basée sur une analyse très fine du contraste dans les images de microscopie électronique à haute résolution sera appliquée au cas des superalliages à base nickel. Cette méthode a été développée par A. Ourmazd et ses collaborateurs [4] pour étudier les variations de composition dans les multicouches de semiconducteurs composés de type AlAs-AlGaAs. La méthode permet, dans les cas les plus favorables, d'obtenir une cartographie chimique avec une résolution proche du niveau atomique.

\section{TECHNIQUES EXPERIMENTALES}

L'alliage étudié est le CMSX2 dont la composition (en pourcentage atomique) est la suivante :

\section{Al 12.5 Ti 1.2 Cr 9 Ni 67.4 Co 5 Ta 2 W 2.5 Mo 0.4}

Après traitement thermique, la phase $\gamma^{\prime}$ se présente sous forme de cuboides, la fraction volumique de ces précipités étant de 60 à 70\%. Les échantillons sont découpés selon les directions [001] et [011]. Ils sont amincis mécaniquement jusqu'à une épaisseur d' environ $100 \mu \mathrm{m}$ puis électrolytiquement pour obtenir une épaisseur compatible avec la haute résolution. Ils sont observés dans un microscope JEOL $4000 \mathrm{EX}$ fonctionnant sous une tension de $400 \mathrm{Kv}$ et dont la résolution est de $0.17 \mathrm{~nm}$ [5]. Le microscope est équipé d'un porte objet goniométrique de double inclinaison à $\pm 20^{\circ}$ ainsi que d'un système d'acquisition et de traitement d'image numérique. Les images expérimentales de haute résolution sont simulées grâce au programme EMS réalisé par P. Stadelmann [6].

\section{ANALYSE CHIMIQUE A HAUTE RESOLUTION : Présentation de la méthode.}

Celle ci a été présentée en détail par ses auteurs [4] et seules les grandes lignes seront rappelées ici. Parmi les faisceaux diffractés par un échantillon cristallin, certains sont extrêmement sensibles à des variations très faibles de composition chimique. Dans un premier temps, ces faisceaux dits chimiques doivent être 
identifiés. Les conditions dans lesquelles leur contribution au contraste de l'image est significative sont alors déterminées. Ces conditions se limitent en général à l'épaisseur $t$ de l'échantillon et la défocalisation $\delta z$ de la lentille objectif du microscope. Leur détermination se fait en calculant la variation de l'intensité des taches de diffraction chimique en fonction de $t$ et par simulation des images correspondantes en fonction de $\delta z$. Les images expérimentales sont alors prises dans des conditions les plus proches possibles des conditions théoriques qui maximisent le contraste chimique et ses variations. La quantification au niveau local de l'information chimique contenue dans les images est réalisée au moyen d'une procédure de reconnaissance de forme. Dans un premier temps, des images témoins d'une cellule élémentaire de chacune des phases pures sont élaborées soit par moyennage à partir des images expérimentales (ce moyennage sur plusieurs cellules élémentaires a pour but de réduire le niveau de bruit ) soit directement à partir des images simulées. Ces témoins sont ensuite digitalisés sur un réseau de $\mathrm{N} \sim 30$ X30 pixels et les intensités correspondant à chaque pixel stockées sur ordinateur. Cet ensemble de valeurs est considéré comme les composantes d'un vecteur dans un espace à $\mathrm{N}$ dimensions. Ainsi chaque phase pure est représentée par un vecteur. Ces deux vecteurs définissent un plan et leur relation angulaire peut être facilement calculée. A travers l'interface séparant les deux phases, la composition varie plus ou moins graduellement. Cette variation peut être représentée grâce à la position angulaire du vecteur intermédiaire lié à chaque cellule élémentaire rencontrée lors du passage de l'interface. Il faut remarquer que ce vecteur intermédiaire ne se trouve pas obligatoirement dans le plan défini par les deux vecteurs représentant les phases pures. Dans ce cas, c'est la composante dans ce plan qui est considérée.

\section{ETUDE DE L'INTERFACE $\gamma / \gamma^{\prime}$}

L'alliage est biphasé. $\gamma$ est une solution solide de structure cubique à faces centrées dans laquelle le nickel est l'élément majoritaire. Pour cette raison, elle sera assimilée à du nickel pur dans les simulations d'images. $\gamma^{\prime}$ possède une structure ordonnée de type $\mathrm{L}_{2} 2$ basée sur le composé Ni3Al. Cette structure ordonnée donne naissance à des taches de surstructure dont l'intensité dépend fortement de la différence entre les facteurs de diffusion atomique des différents éléments chimiques qui portent donc une information chimique. Les deux phases ont des paramètres cristallins très voisins. Les simulations d'images (figure 1) montrent que pour le couple $\mathrm{Ni} / \mathrm{Ni} \mathrm{Al}$, les deux phases présentent un contraste qui permet de les différencier pour une épaisseur supérieure à $10 \mathrm{~nm}$ et une défocalisation $\delta \mathrm{z}=-60 \mathrm{~nm}$. Pour une épaisseur plus faible la tache 100 de surstructure a une intensité très faible et aucun contraste ne différencie les deux phases.

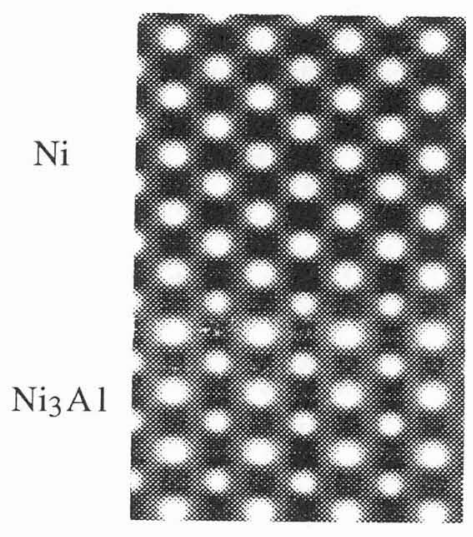

a

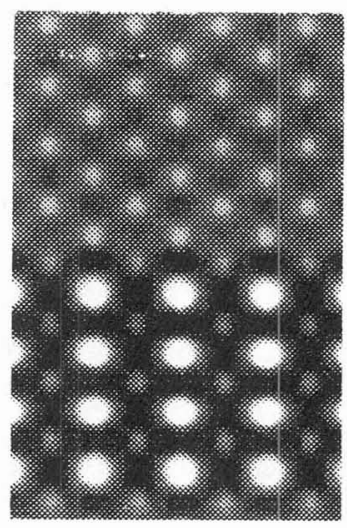

b

Figure 1 : Images simulées d'une interface $\mathrm{Ni} / \mathrm{Ni}_{3} \mathrm{Al}$. Les conditions utilisées sont : $\mathrm{V}=400 \mathrm{kV}$, coefficient d'aberration de sphéricité $\mathrm{Cs}=1.05 \mathrm{~mm}$, divergence du faisceau $0.8 \mathrm{mrad}$, défocalisation chromatique 9 $\mathrm{nm}$.

a épaisseur de l'échantillon $\mathrm{t}=4 \mathrm{~nm}$. défocalisation $\delta \mathrm{z}=-60 \mathrm{~nm}$. Les deux phases présentent un contraste équivalent.

$\mathrm{bt}=12 \mathrm{~nm} . \delta \mathrm{z}=-60 \mathrm{~nm}$. Les deux phases sont nettement différenciées. 

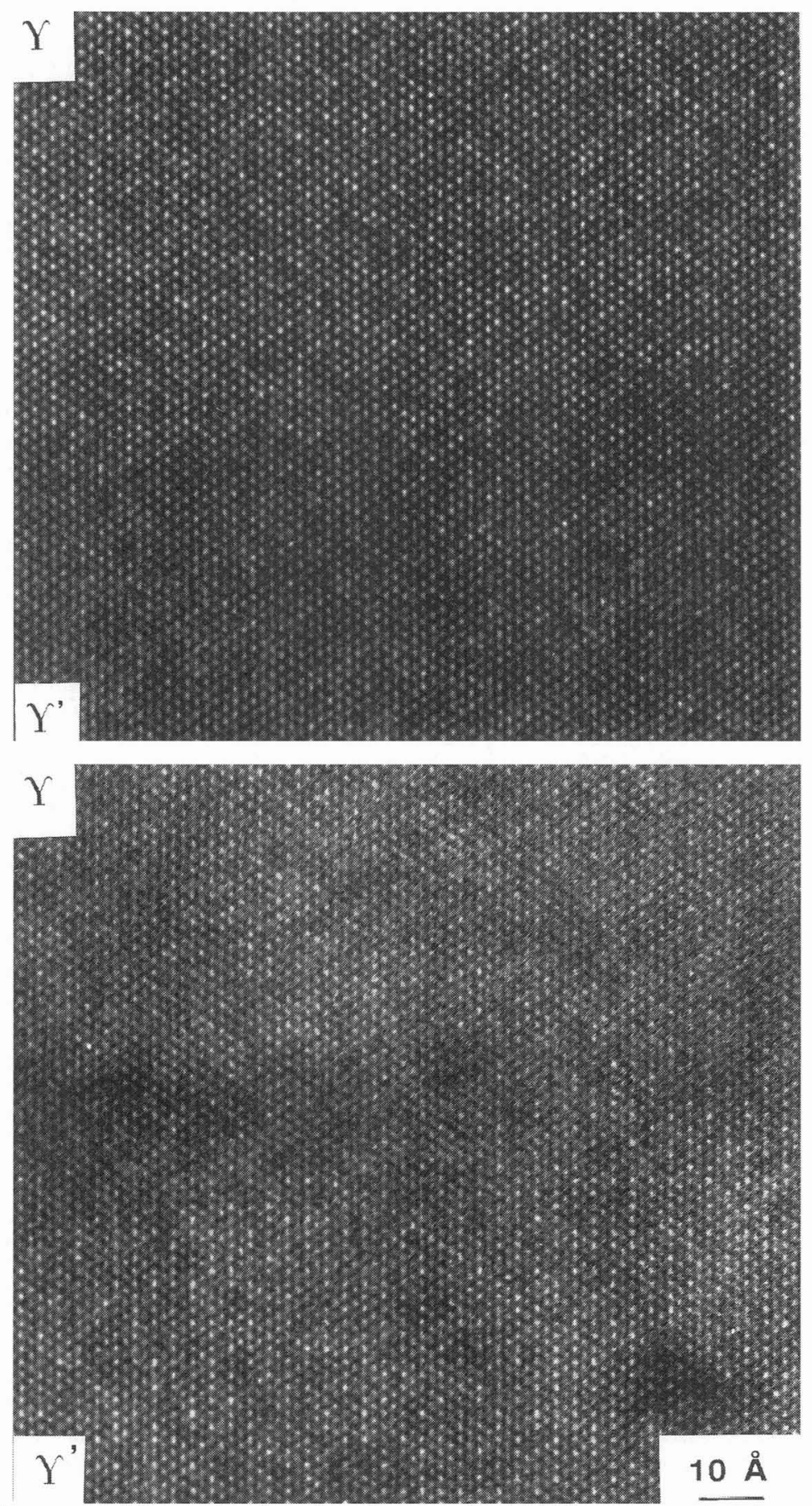

Figure 2 : Images expérimentales d'une interface $\gamma / \gamma^{\prime}(400 \mathrm{kV})$ en orientation [011] a :zone mince : les deux phases sont indiscernables.

$\mathrm{b}:$ zone épaisse : les deux phases $\gamma$ et $\gamma^{\prime}$ ont un contraste différent. 
Les images expérimentales ( figure 2 a et b) prises dans des conditions identiques confirment ce point. Les images expérimentales et simulées ont été analysées selon la procédure exposée dans le paragraphe précédent. La séparation angulaire entre les témoins représentant les deux phases est $\sim 10^{\circ}[7]$. Cette séparation angulaire peut également être exprimée en fonction du rapport signal sur bruit et vaut dans ce cas $\sim 4 \sigma-\sigma$ représentant la largeur moyenne de la distribution des vecteurs expérimentaux autour de leur témoin respectif. La valeur du rapport signal sur bruit est suffisante pour pouvoir séparer les deux phases mais insuffisante pour obtenir une cartographie chimique à l'échelle atomique, une valeur $12 \sigma$ étant nécéssaire ainsi qu'il a été montré dans le cas des interfaces AlAs/ AlGaAs [4]. Cette valeur élevée du bruit est attribuée à la qualité très moyenne des échantillons ainsi qu' au phénomène d'irradiation qui les dégrade assez rapidement.

\section{ETUDE DE LA PHASE $\gamma^{\prime}$}

La phase $\gamma^{\prime}$ a en fait une composition complexe [1]. Pour étudier l'influence de cette composition sur les images de haute résolution des échantillons ont été observés selon la direction [100]. Selon cette orientation en effet les colonnes atomiques projetées ne contiennent qu'un seul élément chimique. Les images expérimentales montrent alors que, dans des conditions bien définies d'épaisseur et de défocalisation, un contraste particulier apparait ( figure $3 \mathrm{a}$ et b). Ainsi, à faible épaisseur $(<10 \mathrm{~nm}$ ), un ensemble de points noirs répartis aléatoirement apparait, alors qu'à épaisseur plus élevée $(\sim 22 \mathrm{~nm})$, un ensemble de points blancs d' intensité supérieure à celle de leurs voisins est présent, cette surintensité peut atteindre $20 \%$ de celle des points environnants. Une analyse précise de la localisation des points blancs surbrillants montre qu'ils ne sont pas répartis au hasard sur tous les sites du réseau cristallin projeté [8]. Ils appartiennent en fait à un sous réseau carré analogue au sous réseau des sites occupés par $\mathrm{Al}$ (figure 4). Ce contraste n'apparait ni sur les images de la solution solide $\gamma$, ni sur celles du composé Ni3Al pur. Ceci suggère donc que le contraste peut être attribué à un effet de composition sur les sites du sous réseau $\mathrm{Al}$ de la structure L12.

La composition de la phase $\gamma^{\prime}$ a été déterminée par Blavette et Bostel [1]. D'après la formule proposée par ces auteurs, il apparait notamment que certains sites atomiques d'aluminium sont partiellement substitués par des atomes lourds tels que : W, Ta, $\mathrm{Ti}$, Ni et $\mathrm{Co}$. Pour tester l'influence de ces atomes lourds sur les images de haute résolution, des images ont été simulées en utilisant une maille de calcul de grande taille dans laquelle certains sites $\mathrm{Al}$ sont partiellement occupés par un atome moyen $\mathrm{X}$ dont la concentration peut varier selon les sites de 0 à $100 \%$. La masse de l'atome moyen X est la moyenne des masses des éléments de substitution pondérée par leur concentration respective. La composition moyenne correspond à la formule simplifiée $\mathrm{Ni}_{3} \mathrm{Al}_{0.6} \mathrm{X}_{0.4}$. La figure 5a représente la maille utilisée pour les simulations d'images. Dans cette maille, la majorité des sites du sous réseau cubique simple de la structure L12 ont une composition moyenne $\mathrm{Al}_{0.6} \mathrm{X}_{0.4}$. Certains de ces sites ont une composition variant entre $\mathrm{Al}$ pur ( site marqué 1.0 ) et $X$ pur ( site marqué .0). Ainsi, la maille de calcul présente toutes les compositions intermédiaires et permet donc d'étudier la variation du contraste en fonction de la composition. Cette méthode utilisant des compositions moyennes ne permet pas d'étudier l'influence de la localisation des atomes lourds X dans l'épaisseur de l'échantillon ( effet "top-bottom" ). En utilisant des conditions d'épaisseur et de défocalisation identiques à celles des images expérimentales, on s'aperçoit que pour certaines compositions des colonnes atomiques, le contraste apparait de façon identique ( figure $5 \mathrm{~b}$ et c).Bien que la variation du potentiel électrique interne varie proportionnellement avec la composition des colonnes, la variation de contraste dans l'image n'est absolument pas linéaire et il est impossible de relier l'intensité à la composition. Ces simulations montrent que pour certaines compositions des colonnes d'aluminium, un contraste apparait mais il ne donne aucune information sur la probabilité d'existence de telles variations locales de composition au sein de l'alliage. Cet effet statistitique a été pris en compte à l'aide d'une loi de distribution binomiale qui donne, pour une colonne d'épaisseur donnée c'est à dire comportant un nombre determiné d'atomes, la probabilité pour qu'elle ait une composition différente de la composition moyenne (figure 6 ). Pour que la substitution partielle des atomes Al, par des atomes lourds donne lieu à un contraste observable, il faut donc que, à la fois la concentration choisie donne effectivement lieu à un contraste et qu'elle ait une probabilité non nulle d'exister : ainsi seul le domaine de composition situé la zone hachurée sur la figure 6 correspond à ces deux conditions simultanées. 


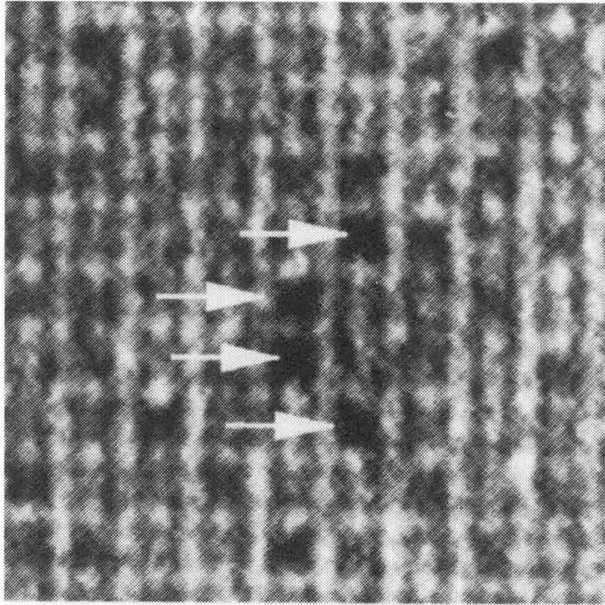

a

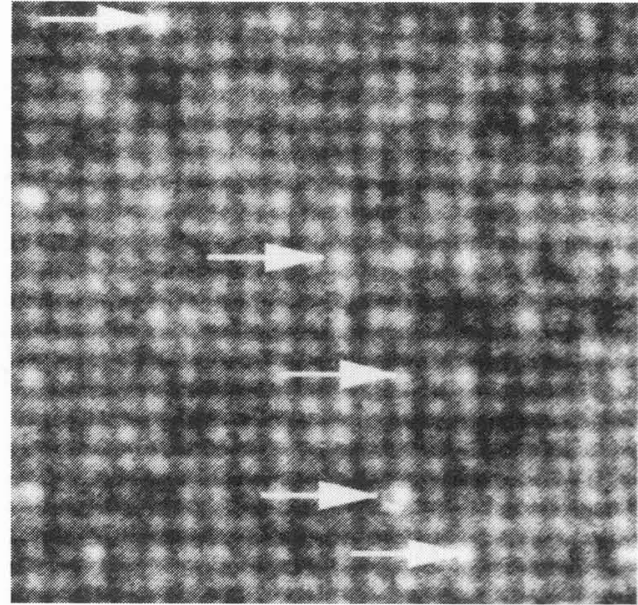

b

Figure 3 : Images expérimentales de la phase $\gamma^{\prime}$ selon l'orientation [001].

- zone mince de l'échantillon. Un fort contraste de points noirs apparait sur certaines colonnes.

- zone épaisse. caractérisée par un contraste de points blancs surbrillants.

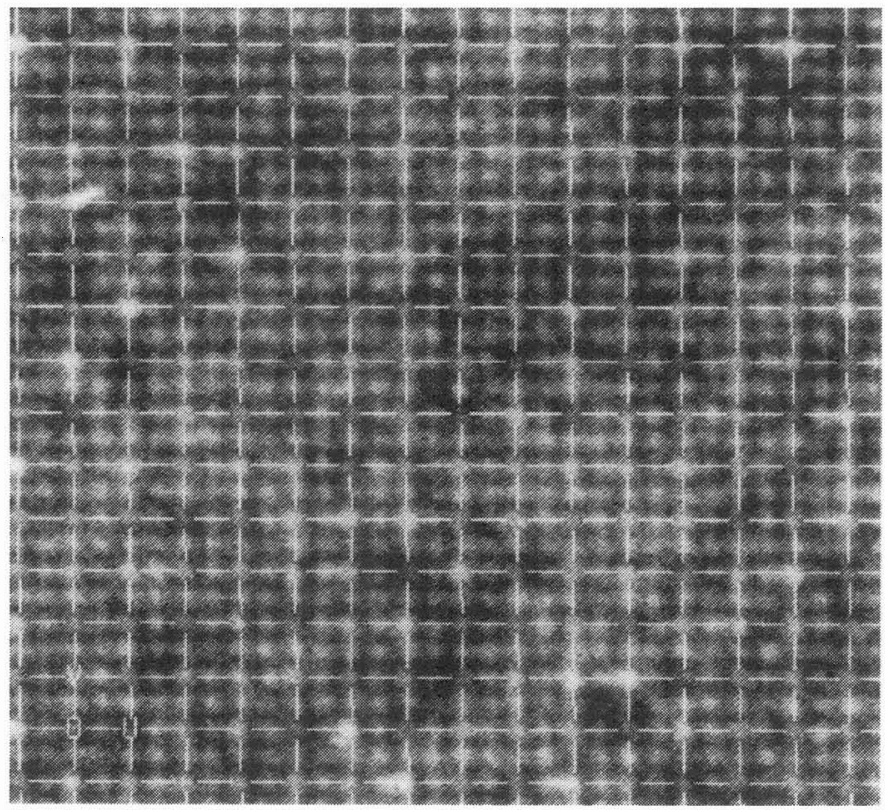

Figure 4 : Mise en évidence de la localisation des points surbrillants sur le sous réseau des positions atomiques de l'aluminium. 


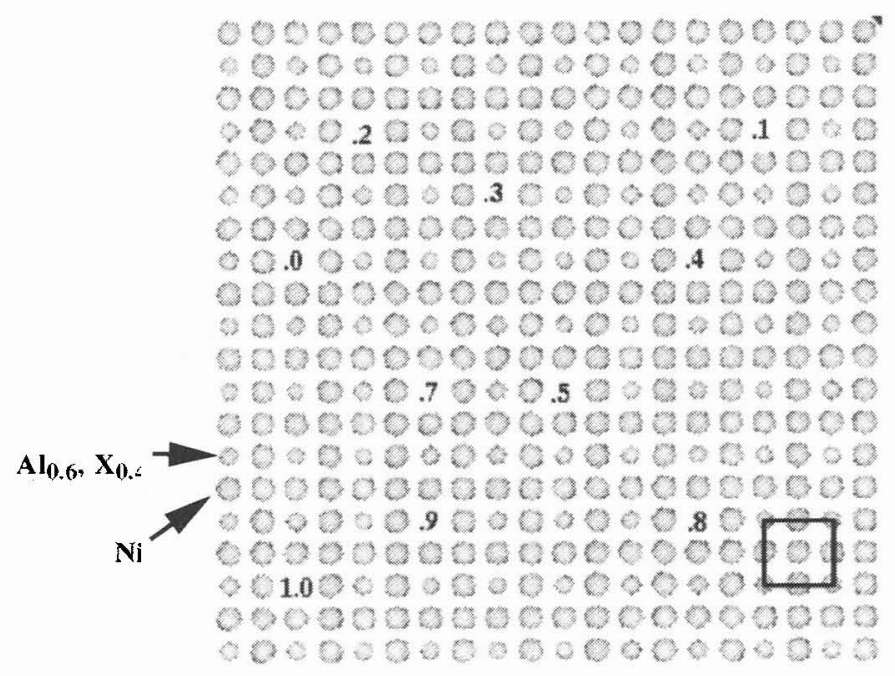

a

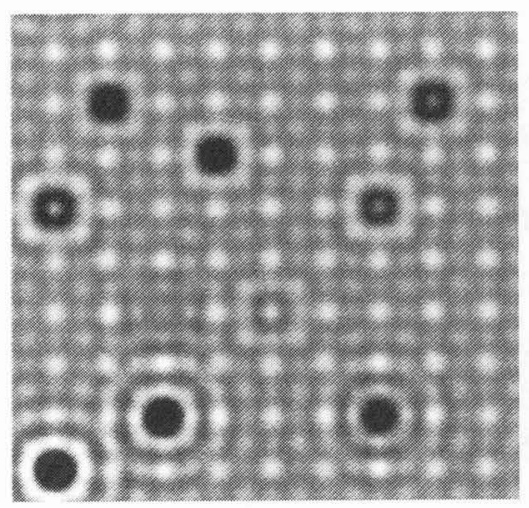

b

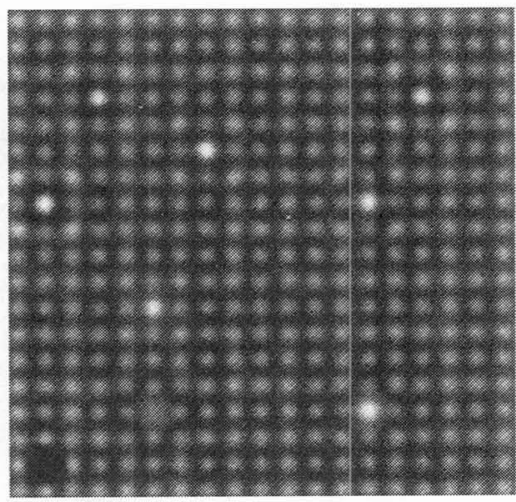

$c$

Figure 5 -a Maille de calcul utilisee pour les simulations (projection[001]). Les nombres donnent la proportion en aluminium dans les colonnes.

- b epaisseur $\mathrm{t}=14 \mathrm{~nm}$ defocalisation $\delta \mathrm{z}=-30 \mathrm{~nm}$. Pour certaines compositions, les colonnes correspondantes montrent un fort contraste noir

$-c t=21.5 \mathrm{~nm} \delta=-30 \mathrm{~nm}$. Un contraste de points blancs surbrillants est présent. 


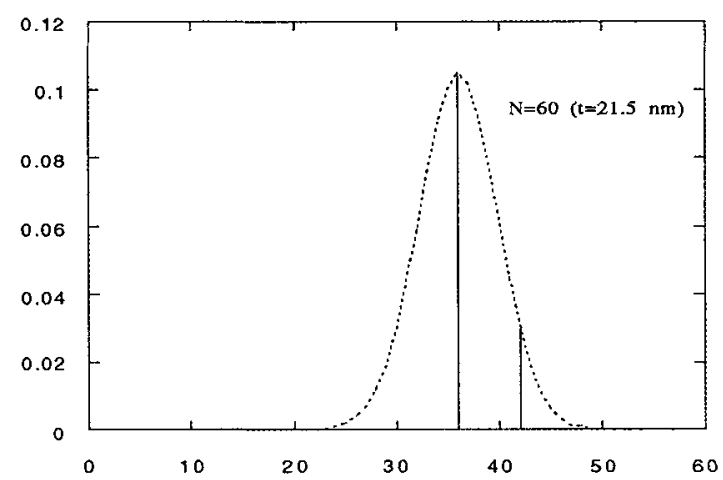

Figure 6 : Probabilité d'existence de fluctuations de composition dans une colonne atomique contenant 60 atomes (correspondant à une épaisseur de $21.5 \mathrm{~nm}$ ). La distribution est centrée sur la valeur moyenne ( $c=0.6$ soit 36 atomes). Seules les compositions situées dans la zone hachurée donnent lieu à un contraste observable sur les images.

\section{CONCLUSION}

La technique d'analyse chimique par microscopie électronique à haute résolution qui a été brièvement exposée permet de donner des renseignements sur la composition locale dans des échantillons métalliques. Les résultats sont pour le moment moins précis que dans l'étude des interfaces dans les systèmes semiconducteurs. Ceci tient à plusieurs causes différentes. Les paramètres cristallins sont nettement plus petits dans les composés métalliques : la qualité des images s'en ressent par une diminution très nette du rapport signal sur bruit. Dans le système étudié, les taches de diffraction chimique sont moins sensibles aux variations de composition et ceci d' autant moins que les sites Al de la phase $\gamma^{\prime}$ sont partiellement substitués par des atomes lourds. La méthode apporte néamoins des informations sur la composition locale.

\section{Réferences :}

[1] D. Blavette and A. Bostel Acta Met. 32(1984) 811.

[2] A. Chiba, D. Shindo and S. Hanada Acta Met. Mater. 39 (1991) 13.

[3] O. Krivanek, A. J. Gubbens, N. Delby and C. E. Meyer Microsc. Microanal. and Microstruct. 3 (1992) 187.

[4] A. Ourmazd, F. H. Baumann, M. Bode and Y. Kim Ultramicrosc. 34 (1990) 237.

[5] A. Bourret and J. M. Pénisson JEOL News 25E (1987) 2.

[6] P. Stadelmann Ultramicrosc. 21 (1987) 131.

[7] J. M. Pénisson, M. Bode, F.H. Baumann and A. Ourmazd Phil. Mag. Lett. 64 (1991) 269.

[8] J. M. Pénisson Ultramicrosc. 51 (1993) 264. 